\title{
SUBARU/FOCAS Globular Clusters Survey around M82
}

\author{
Yoshihiko Saito, Masanori Iye, Nobunari Kashikawa, Koji S. Kawabata \\ National Astronomical Observatory Japan (NAOJ), 2-21-1, Osawa, \\ Mitaka, Tokyo, 181-8588 Japan \\ Michitoshi Yoshida \\ Okayama Astrophysical Observatory, NAOJ, Kamogata, Okayama, \\ 719-0232 Japan
}

Youichi Ohyama, Toshiyuki Sasaki, Tadafumi Takata, George Kosugi, Kentaro Aoki

Subaru Telescope, NAOJ, 650 North A'ohoku Place, Hilo, Hawaii, 96720 USA

\section{Motivation}

Recently, a number of possible young globular clusters (GCs) have been found in some merging/interacting galaxies or starburst galaxies. On the other hand, it is well known that GCs are numerous in giant elliptical galaxies many of which are thought to be formed via major merger of galaxies. These facts lead us to a thought that the formation process of GCs could accompany galaxy - galaxy interaction followed by starburst. The archetypical starburst galaxy M82 is an ideal target to study the relation between galaxy interaction, starburst, and GC formation.

\section{Summary}

We carried out search of GCs in M82, by taking advantage of high-resolution imaging and deep spectroscopic capabilities provided by FOCAS on the Subaru telescope. We applied a scheme for searching for GCs in disk/irregular galaxies that combines color selection, spectral analysis, and profile analysis for identifying GCs. Especially, the profile analysis is a key method for this scheme. We cannot identify GCs only from color selection and spectral analysis around nearby galaxies having low receding velocity, because we cannot distinguish late-F - early-G type stars in our Galaxy from those GCs. In the central 6 arc-minutes diameter region of M82, we found two bona fide GCs and 8 young GC candidates in our first attempt at searching for GCs in M82. The estimated total number of bona fide GCs is about 10 corresponding to the expected number from the initial luminosity of M82. We conclude that M82 has a GC system similar to our Galactic specific frequency before the interaction, and newly born GCs should be formed by the tidal interaction with M81. 